\title{
Fatores de Correção para Ganho de Peso Médio Diário no Período do Nascimento ao Desmame em Bovinos da Raça Nelore ${ }^{1}$
}

\author{
Claudia Cristina Paro de Paz ${ }^{2,3}$, Lúcia Galvão de Albuquerque ${ }^{3,4}$, Luiz Alberto Fries $3,5,6$
}

\begin{abstract}
RESUMO - O objetivo deste trabalho foi calcular fatores de correção para os efeitos da idade da vaca (IDV), idade do bezerro (IDB) e data de nascimento juliana (DJN) sobre ganho de peso médio diário (GMD) no periodo do nascimento ao desmame de animais da raça Nelore. Os dados foram analisados por máxima verossimilhança restrita, empregado-se modelo animal. O modelo incluiu os efeitos fixos de grupo contemporâneo (GC), as covariáveis idade da vaca, idade do bezerro e data juliana de nascimento e os efeitos aleatórios direto (animal), materno, ambiente permanente e resíduo. Os dados foram submetidos à análise prévia para definição dos polinômios segmentados a serem usados, empregando-se o programa computacional SAS, no qual foram criados os conjuntos de covariáveis. No estudo do GMD, em função de IDV, foi observado efeito quadrático-quadrático, e as vacas atingiram a maturidade entre o $5^{\circ}$ e $7^{\circ}$ ano de idade. O GMD predito para os bezerros aumentou para as mães no período do $4^{\circ}$ ao $6^{\circ}$ ano de idade, em que se atingiu o ganho máximo, decrescendo dos 6 até os 15 anos de idade, quando os bezerros apresentaram a menor taxa de ganho. Para IDB, foi observado efeito quadrático simples. O GMD apresentou média geral de $0,681 \mathrm{~kg} /$ dia e foi sempre decrescente à medida que aumentou a idade do animal (entre 156 a 308 dias de idade). O efeito da data de nascimento juliana, apresentou efeito linear sobre GMD, que diminuiu no período de 220 até 365 dias do calendário juliano. Estes dois efeitos (IDB e DJN) foram os mais difíceis de modelar: os nós e os segmentos dos polinômios obtidos a cada passo foram dependentes e/ou influenciados pela modelagem usada para os outros efeitos, sugerindo problemas de multicolinearidade e, ou concentração de dados em pontos de influência. Em função destes problemas, optou-se por modelagem simplificada dos efeitos IDB e DJN.
\end{abstract}

Palavras-chave: data juliana de nascimento, grupo contemporâneo, idade do bezerro ao desmame, idade da vaca ao desmame

\section{Correction Factors for Average Daily Gain from the Birth to Weaning Period in Nellore Breed Cattle}

\begin{abstract}
The objective of this work was to calculate correction factors for the effects of age of the dam (AOD), age of the calf (AOC) and Julian birth date (DOB) on the average daily gain (ADG) from birth to weaning period of animals of Nellore breed. The data were analyzed by restricted maximum likelihood, using the animal model. The model included the fixed effects of contemporary group (CG), AOD, AOC and DOB, and the direct effects (animal), maternal, permanent environmental and residue. The data were submitted to previous analysis for the definition of segmented polynomials to be used by means of SAS computing program where the covariates sets were created. In the ADG study in function of AOD, was observed a quadractic-quadractic effect, and the dams reached the maturity between the $5^{\text {th }}$ and $7^{\text {th }}$ years of age. The predicted ADG for the calves increased for the dams in the $4^{\text {th }}$ and $6^{\text {th }}$ years of age, as that the maximum gain was reached, decreasing from 6 up to 15 years of age, when the calves presented the lower gain rate. For AOC was observed a simple quadratic effect. The ADG presented an overall mean of .681 kg/day and decreased as the animal age increased (from $156^{\text {th }}$ to $308^{\text {th }}$ day of age). The Julian birth date showed a linear effect on the ADG, that decreased in the period from $220^{\text {th }}$ to $365^{\text {th }}$ day of the Julian calendar. These two effects (AOC and DOB) were the most difficult to model: the "knots" and the segments of obtained polynomials at each step were dependent and, or influenced by the used modeling for the other effects, suggesting multicolinearity problems and, or concentration of data in influent points. In function of these problems, a simplified modeling of the AOC and DOB effects was choose.
\end{abstract}

Key Words: Julian births date, contemporaneous groups, calf age at weaning, , dam age of weaning

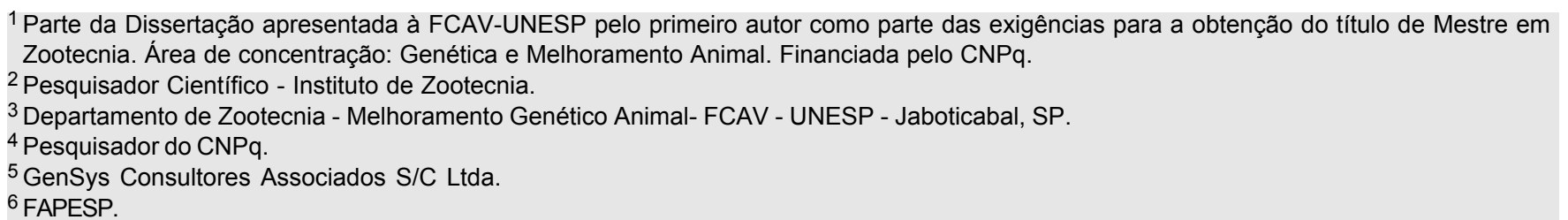




\section{Introdução}

As características de crescimento no período do nascimento ao desmame são de grande importância econômica e fácil mensuração e servem para avaliar o potencial genético de crescimento do bezerro, além da produção leiteira da vaca.

Estas características são influenciadas por diversos efeitos de ambiente, destacando-se sexo, mês e ano de nascimento, idade da vaca e idade do bezerro. Trabalhos mais recentes têm encontrado efeito significativo da data de nascimento do animal sobre as características de crescimento pré-desmame, mostrando que fatores de correção para a data de nascimento são mais apropriados que a simples inclusão do mês de nascimento no modelo de análise (CAMPOS et al., 1989; LUCERO et al., 1995).

Efeitos significativos de idade da vaca e idade do bezerro sobre os pesos e ganhos de pesos no período pré-desmame têm sido descritos em diversos trabalhos realizados no Brasil e em outros países, com raças européias (AHUNU e MAKARECHIAN 1986, 1987; CARDELINO e CASTRO, 1987; ELZO et al., 1987; EUCLIDES FILHO et al., 1991; PEIXOTO, 1993; SOUZA et al., 1994; e ANDRADE et al., 1994).

A idade da vaca é fator de grande importância que deve ser levado em consideração nos programas de seleção e manejo dos rebanhos de corte. Em geral, as vacas de primeira cria desmamam bezerros de 10 a $15 \%$ mais leves que as vacas adultas. A presença de interações com raça, sexo e nível nutricional dos bezerros dificulta as estimativas deste efeito.

A maioria dos trabalhos encontrados na literatura considera que a idade do bezerro apresenta efeito linear sobre as características de peso e ganho de peso pré-desmame, ao passo que, para a idade da vaca, considera efeito quadrático sobre estas características, sendo os fatores de correção estimados para determinado conjunto de dados apropriados para todos os rebanhos, em todos os anos. Quanto à data de nascimento, apenas inclui-se no modelo de análise o mês de nascimento, seguindo as recomendações da Beef Improvement Federation (PAZ, 1997).

No entanto, alguns trabalhos mostraram efeito não-linear da idade do bezerro ao desmame e a existência do efeito de estacionalidade dentro da estação de nascimento, mostrando que fatores de correção para a data de nascimento são mais apropriados que a simples inclusão do mês de nascimento no modelo de análise (FRIES, 1984; CAMPOS et al., 1989; e LUCERO et al., 1995).
O objetivo do presente trabalho foi estimar fatores de correção para os efeitos de idade da vaca (IDV), idade do bezerro ao desmame (IDB) e data juliana de nascimento (DJN), sobre o ganho médio diário de peso pré-desmame (GMD) em bovinos de corte da raça Nelore.

\section{Material e Métodos}

Foram utilizados dados de ganho de peso médio diário no período do nascimento ao desmame de 18.772 bezerros da raça Nelore nascidos entre 1984 e 1994. Os registros de produção usados no presente estudo foram obtidos junto a GenSys Consultores Associados S/C Ltda e pertencem à Granja Rezende S.A., que se dedica a atividades agropecuárias em Uberlândia, Minas Gerais, realizando seleção nos seus rebanhos. A seleção é efetuada com base no desempenho e na fertilidade dos animais.

A edição dos dados foi realizada de forma a serem criadas as variáveis IDB, IDV e DJN como variáveis classificatórias (IDB variou de 15 a 30; IDV, de 4 a 15; e DJN, de 10 a 18), o ganho de peso do nascimento ao desmame não ajustado (GND) e o GMD. Assim, foram efetuados os testes dos ajustes prévios e também verificou-se o comportamento das funções do ganho de peso pré-desmame em função de IDB, IDV e DJN, para posterior modelagem pelos Polinômios Segmentados e Ordinários. Em seguida, criou-se a variável grupo contemporâneo pela concatenação das variáveis sexo, fazenda, retiro, manejo, data juliana de desmame e ano de nascimento do bezerro. Os animais nascidos no outono foram eliminados, devido ao pequeno número de animais. Os animais cruzados também foram eliminados, restando apenas os animais puros PO e PC. Eliminaramse os animais cujas mães tinham menos de 4 anos e mais de 15 anos. A eliminação das novilhas (mães com 3 anos de idade) ocorreu em virtude das diferenças existentes nas práticas de manejo realizadas na fazenda, exclusivamente com estes animais, sem que estas diferenças estivessem codificadas no arquivo, portanto, não podendo ser detectadas, o que poderia alterar os resultados. Os animais cujas mães tinham mais de 15 anos foram eliminados simplesmente pelo pequeno número de observações. Submeteu-se o arquivo à contagem do número de filhos por touro e, em seguida, à contagem do número de observações por grupo contemporâneo, eliminando-se os animais, cujos touros tinham menos de 4 filhos e os grupos contemporâneos com menos de 4 animais, resultando em 18.772 animais, filhos de 7619 vacas e 109 touros. 
As pré-correções dos dados de ganho total de peso pré-desmame que vêm sendo efetuadas pela Granja Rezende S.A., para os efeitos de IDV, IDB e DJN, foram testadas com o objetivo de verificar se os dados estavam sendo bem ajustados. Para efetuar este teste, foi realizada estimação dos efeitos da idade da vaca, idade do bezerro ao desmame e data juliana de nascimento sobre o ganho em peso prédesmame ajustado (GNDA), pelas análises de variância, utilizando-se o método dos quadrados mínimos, por intermédio do procedimento GLM (SAS, 1992), utilizando-se modelo com os seguintes efeitos fixos: grupo contemporâneo, idade da vaca, idade do bezerro ao desmame e data juliana de nascimento.

Os Polinômios Segmentados têm sido utilizados em estudos de curvas de crescimento de bovinos de corte e aves, curvas de produção de leite e ovos e para a estimação de fatores de correção para os efeitos fixos. As funções polinomiais estudadas no presente trabalho, na tentativa de modelar as curvas para os efeitos IDV, IDB e DJN dentro da estação, sobre o GMD, foram descritas em PAZ (1997).

Para a modelagem dos efeitos de ambiente foram realizadas as seguintes etapas: (1) os efeitos IDV, IDB e DJN foram incluídos em um modelo fixo, como variáveis discretas (para IDB e DJN foram utilizadas classes de 10 e 20 dias, respectivamente) juntamente com GC; (2) por inspeção dos diagramas de pontos das soluções obtidas, diferentes polinômios segmentados foram testados e os nós foram determinados com base nos quadrados médios dos resíduos; (3) o modelo final foi determinado por ponderação de testes estatísticos, magnitude dos efeitos e capacidade de explicação biológica. As etapas seguidas para a modelagem dos efeitos ambientais estão descritas em PAZ (1997).

A idade da vaca foi modelada como efeito Quadrático-Quadrático, com o ponto de junção ("nó") aos 8 anos de idade (para machos e fêmeas). A idade do bezerro foi descrita por regressão quadrática simples (Polinômio Ordinário). A data de nascimento dentro do calendário juliano foi descrita por meio de regressão linear simples (Polinômio Ordinário).

O maior obstáculo para o uso de Polinômios Segmentados é que os "nós" ou pontos de junção devem ser conhecidos. O conhecimento prévio na área técnica específica, o uso de diagramas e as médias para a estimação por inspeção dos "nós" ou o uso de um modelo não-linear para a estimação simultânea dos "nós" e dos coeficientes de regressão podem ser empregados nesta tarefa (FULLER, 1969).
A determinação da posição dos "nós" e dos coeficientes de regressão de cada segmento da curva e a técnica de utilização dos polinômios segmentados são descritos em PAZ (1997).

\section{Cálculo dos fatores de correção}

A BIF (1996) recomenda, para o ajuste dos pesos em gado de corte, fatores de correção aditivos, para as condições dos Estados Unidos, em que a variação dos registros de produção é pequena.

Em gado de leite, fatores de correção multiplicativos têm sido preferidos aos fatores aditivos, por causa da variação dos registros de produção de leite que estão intimamente relacionados com a média do rebanho, assim, o aumento em produção com a idade tende a ser maior em rebanhos de alta produção. Dessa forma, a utilização de fatores multiplicativos, quando se trabalha com grandes variações nas produções dos rebanhos, apresenta menores vícios quando comparados aos fatores aditivos (MILLER, 1973).

No presente estudo o ganho médio diário de peso pré-desmame apresentou grande variação (variou de 0,2690 a 1,3840 kg/dia). Assim, neste estudo foram preferidos os fatores de correção multiplicativos.

Os fatores de correção foram calculados utilizando-se as soluções obtidas para cada efeitode acordo com omodelo:

em que

$$
\mathrm{y}=\mathrm{X} \beta+\mathrm{Za}+\mathrm{Mm}+\mathrm{Wp}+\mathrm{e}
$$

$\mathrm{y}=$ Vetor da variável dependente (GMD);

$\mathrm{X}=$ Matriz de incidência de efeitos fixos;

$\beta=$ Vetor dos efeitos fixos (grupo contemporâneo, como variável classificatória e as covariáveis idade da vaca ao parto e do bezerro ao desmame e data juliana de nascimento);

$Z=$ Matriz de incidência de efeitos genéticos aditivos diretos;

$\mathrm{a}=$ Vetor de valores genéticos aditivos diretos dos animais;

$M=$ Matriz de incidência dos efeitos genéticos aditivos maternos;

$\mathrm{m}=$ Vetor de valores genéticos aditivos maternos;

$\mathrm{W}=$ Matriz de incidência do efeito de ambiente permanente da vaca;

$\mathrm{p}=$ Vetor de efeito de ambiente permanente da vaca; e $\mathrm{e}=$ Vetor de efeitos residuais aleatórios.

$$
E=\left[\begin{array}{c}
\beta \\
a \\
m \\
p \\
e
\end{array}\right]=\left[\begin{array}{l}
\beta \\
0 \\
0 \\
0 \\
0
\end{array}\right] ; \quad \operatorname{Var}\left[\begin{array}{l}
a \\
m \\
p \\
e
\end{array}\right]=\left[\begin{array}{cccc}
\sigma_{a}^{2} A & \sigma_{a m} A & 0 & 0 \\
\sigma_{a m} A & \sigma_{m}^{2} A & 0 & 0 \\
0 & 0 & \sigma_{p}^{2} I_{C} & 0 \\
0 & 0 & 0 & \sigma_{e}^{2} I_{N}
\end{array}\right]
$$

Nestas análises, foram geradas total de 58243 equações de modelos mistos. A idade da vaca foi considera- 
da em anos e a idade do bezerro e a data juliana de nascimento, em dias. O número de níveis para o efeito de grupo contemporâneo foi de 613; o número de vacas, de 7619; o número de touros, de 109; o número de animais que tiveram seus desempenhos medidos, de 18.772; o número de animais na matriz de parentesco, de 25.001; e o número de covariáveis, 9.

Os fatores de correção foram calculados utilizandose as soluções obtidas para cada efeito de acordo com este modelo anterior, de acordo com a seguinte fórmula:

em que

$$
\mathrm{FC}_{\mathrm{X}}=\text { gmd }_{\text {base }} / \text { gmd }_{\mathrm{X}}
$$

$\mathrm{FC}_{\mathrm{X}}=$ fator de correção para o ponto $\mathrm{X}$ em função da base;

gmd $_{\text {base }}=$ ganho médio diário de peso pré-desmame estimado na base; e

$\operatorname{gmd}_{\mathrm{X}}=$ ganho médio diário de peso pré-desmame estimado no ponto $\mathrm{X}$.

\section{Resultados e Discussão}

O GND deste rebanho, pertencente à Granja Rezende S.A., vem sendo ajustado por fatores de correção obtidos por CAMPOS et al. (1989). A Tabela 1 mostra o resumo das análises de variância do ganho de peso pré-desmame ajustado. Verificou-se que todos os efeitos ajustados (idade da vaca, idade do bezerro e data de nascimento) continuavam apresentando influência significativa sobre GNDA, indicando que nenhum destes efeitos estavam sendo ajustados completamente. Este resultado confirma a necessidade de estimação de fatores de correção específicos para cada população, como já relatado por Northcutt e Wilson (1994), citados e descritos por WOLF (1996).

Northcutt e Wilson (1994), citados por WOLF (1996), verificaram mudanças nos valores dos fatores de correção recomendados pela BeefImprovement Federation (BIF), para o efeito da idade da vaca sobre o ganho de peso pré-desmame. Da mesma forma, WOLF (1996) observou que, mesmo tendo sido ajustado para fatores obtidos em outro conjunto de dados, o ganho de peso pré-desmame apresentou significância dos efeitos para os quais ajustes haviam sido feitos (idade da vaca, idade do bezerro e data de nascimento).

As mudanças observadas nos fatores de correção podem ser atribuídas às modificações que ocorreram com o rebanho, com relação aos fatores não-genéticos, como alterações no manejo ou no ambiente em que se desenvolvem os animais. Nota-se que CAMPOS et al. (1989) obtiveram os fatores de correção em 1989 com dados de Nelore, mas de outras fazen- das situadas em outros Estados do país.

Idade da vaca

Os coeficientes de regressão encontrados foram: $0,02098985 \mathrm{~kg} / \mathrm{dia} ;-0,00169434 \mathrm{~kg} / \mathrm{dia}^{2} ;$ e 0,00121417 $\mathrm{kg} / \mathrm{dia}^{2}$ para machos e $0,00306791 \mathrm{~kg} / \mathrm{dia} ;-0,00025541$ $\mathrm{kg} / \mathrm{dia}^{2}$; e $-0,00077867 \mathrm{~kg} / \mathrm{dia}^{2}$ para fêmeas, para coeficiente de regressão linear, primeiro quadrático e a diferença de quadratura entre o primeiro e o segundo segmento quadrático, respectivamente. A idade da vaca variou de 4 a 15 anos.

As equações estimadas para o efeito da idade da vaca ao parto, sobre GMD de machos e fêmeas, foram: $\hat{Y}_{I D V(\text { machos })}=0,6598137+0,02098985 I D V-0,00169434 I D V^{2}+0,00121417 Z$; $\hat{Y}_{I D V(\text { femeas })}=0,6318004+0,00306791 I D V-0,00025541 I D V^{2}-0,00077867 Z$;

em que: $Z=0$, se $\operatorname{IDV}<8$; e $Z=(\operatorname{IDV}-8)^{2}$, se IDV $>8$.

$\hat{Y}=$ Ganho médio diário de peso pré-desmame predito O primeiro segmento quadrático apresentou ponto de máximo para machos e fêmeas, pois $\mathrm{a}^{2}$ foi negativo para ambos. As vacas atingiram maturidade entre 5 e 7 anos de idade e o GMD predito para os bezerros variou de 0,65293 a $0,72476 \mathrm{~kg} /$ dia para machos e 0,5822 a $0,64101 \mathrm{~kg} /$ dia para fêmeas; aumentou no período de $4(0,7166 \mathrm{~kg} / \mathrm{dia}$ para machos e 0,63999 $\mathrm{kg} /$ dia para fêmeas) a 6 anos, no qual atingiu o ganho máximo (tanto para machos quanto para fêmeas), decrescendo depois até os 15 anos anos, em que ocorreu a menor taxa de ganho de peso (Figura 1).

CARDELINO e CASTRO (1987) encontraram efeito quadrático (Polinômio Ordinário) para a idade da vaca sobre o peso ao desmame e o ganho de peso pré-desmame, que aumentaram até 6-9 anos (idade da vaca); após esta idade, ocorreu declínio dos pesos e ganhos de pesos dos bezerros, para os bezerros filhos de vacas adultas.

FRIES (1984), CAMPOS et al. (1989) e LUCERO et al. (1995), utilizando Polinômios Segmentados, observaram sistema quadrático-quadrático para o efeito da idade da vaca sobre o ganho de peso pré-desmame.

A Figura 1, que apresenta as curvas para o GMD em função da idade da vaca ao parto de machos e fêmeas, indica a existência de interação entre os efeitos de sexo e idade da vaca ao parto. O GMD atingiu o pico aos seis anos de idade da vaca ao parto, tanto para machos quanto para fêmeas. Entretanto, os machos apresentaram aumento da taxa de ganho de peso pré-desmame, dos 4 aos 6 anos de idade, mais acentuado que as fêmeas. Praticamente o GMD nas fêmeas apresentou platô dos 4 aos 8 anos de IDV. 
Rev. bras. zootec.

Tabela 1- Fontes de variação, graus de liberdade e quadrado médio do ganho de peso pré-desmame ajustado (GNDA)

Table 1- Sources of variation, degrees of freedon and mean square for preweaning adjusted weight gain

\begin{tabular}{lcc}
\hline $\begin{array}{l}\text { Fontes de variação } \\
\text { Source of variation }\end{array}$ & $\begin{array}{c}\text { Graus de liberdade } \\
\text { Degree of freedon }\end{array}$ & $\begin{array}{c}\text { Quadrado médio(GNDA) } \\
\text { Mean square }\end{array}$ \\
\hline $\begin{array}{l}\text { Grupo contemporâneo } \\
\text { Contemporary group }\end{array}$ & 612 & $7475,05^{* *}$ \\
$\begin{array}{l}\text { Classe de idade da vaca } \\
\text { Age of dam }\end{array}$ & 11 & $3670,54^{* *}$ \\
$\begin{array}{l}\text { Classe da idade do bezerro } \\
\text { Age of calf (classes) }\end{array}$ & 15 & $4791,05^{* *}$ \\
$\begin{array}{l}\text { Classe de data de nascimento } \\
\text { Birth date (classes) }\end{array}$ & 7 & $1001,25^{*}$ \\
$\begin{array}{l}\text { Resíduo } \\
\text { Residual }\end{array}$ & 18126 & 350,33 \\
$\mathrm{R}^{2}(\%)$ & & 52,96 \\
${ }^{*} \mathrm{P}<0,05 ;{ }^{* *} \mathrm{P}<0,01$. & &
\end{tabular}

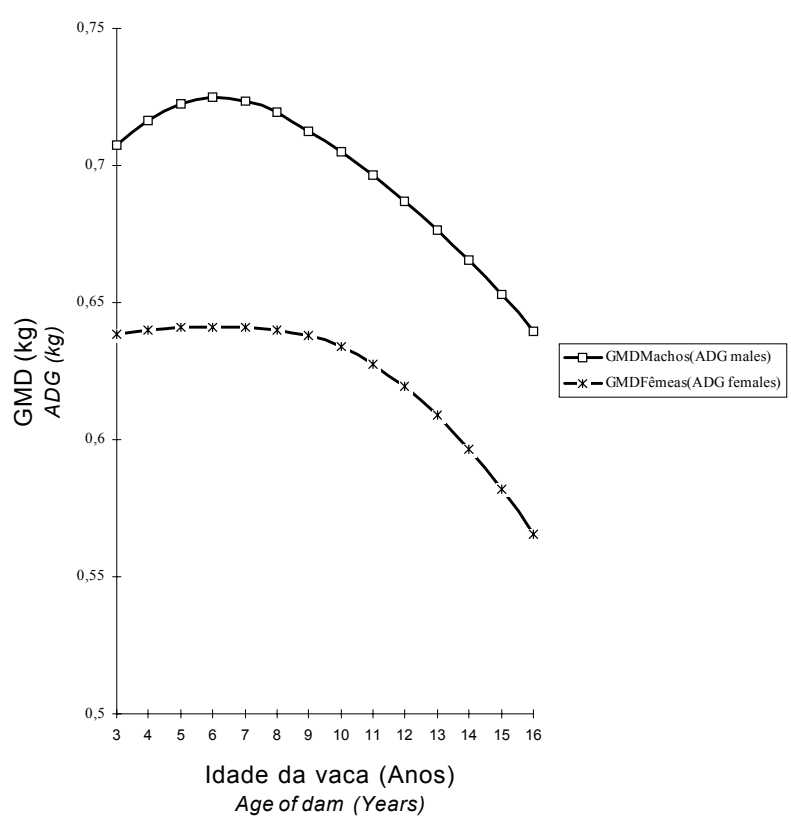

Figura 1 - Curva estimada pelo Polinômio Segmentado Quadrático-Quadrático para o ganho médio diário de peso pré-desmame de machos e fêmeas, de acordo com a idade da vaca ao parto.

Figure 1 - Estimate curve by quadractic-quadractic segmented polynomial for preweaning average daily gain of males and females, in accordance with the age of dam at calving.

Estas diferenças entre machos e fêmeas são ainda maiores com relação aos pesos do animais. Provavelmente, o que pode estar ocorrendo é que os machos, por apresentarem potencial de crescimento diferenciado das fêmeas, aproveitam melhor o pico de produção de leite de suas mães, que é atingido em torno dos seis anos de idade, o que não acontece com as fêmeas.

Assim, como recomendação prática, as novilhas (três anos), por sofrerem maior desgaste para criar seus bezerros, poderiam ser manejadas de maneira diferenciada, dependendo do sexo de seus filhos. Novilhas mães de bezerros machos poderiam receber suplementação alimentar na tentativa de compensar este maior desgaste, ou pelo menos, as lactações poderiam sofrer reajuste em função disto. Esta é uma das vantagens de se realizarem grupos contemporâneos separados para machos e fêmeas.

Os fatores de ajuste para IDV de machos e fêmeas foram calculados considerando como base oito anos de idade da vaca ao parto (Tabela 2).

Os fatores de correção para o efeito da idade da vaca sobre o ganho médio diário de peso pré-desmame variaram de 0,99246 a 1,10164, para machos, e de 0,99842 a 1,09928, para fêmeas, mostrando diferenças de até $10 \%$ nos fatores de correção para idade da vaca ao parto em machos e de até $9,9 \%$ em fêmeas.

Idade do bezerro e data de nascimento

Os fatores de ajuste para IDB e DJN foram calculados considerando, como base, 240 dias de idade do bezerro ao desmame e 300 dias de data juliana de nascimento.

O ganho médio diário apresentou média geral observada de $0,681 \mathrm{~kg} /$ dia variando de acordo com a idade do animal de 0,849 a $0,611 \mathrm{~kg} /$ dia (médias das classes $15^{\mathrm{a}}$ e $30^{\mathrm{a}}$, respectivamente) no intervalo de 156 a 308 dias. O ganho de peso médio diário foi sempre decrescente à medida que aumentou a idade do animal, sendo que a queda foi mais acentuada entre 156 e 200 dias; deste ponto, até 308 dias, a taxa 
PAZ et al.

\begin{tabular}{|c|c|c|c|c|}
\hline Table 2 - & Average daily gain ( $A D$ & nd correction factors for preweanin & daily gain according & fdam (AD) formales and females \\
\hline$\overline{\mathrm{IDV}}$ & GMD (machos) & Fator de correção (machos) & GMD (fêmeas) & Fator de correção (fêmeas) \\
\hline$A D$ & $A D G$ (males) & Correction factor (males) & $A D G$ (females) & Correction factor (females) \\
\hline$\overline{04}$ & 0,71660 & 1,00367 & 0,63999 & 1,00002 \\
\hline 05 & 0,72240 & 0,99570 & 0,64075 & 0,99882 \\
\hline 06 & 0,72476 & 0,99246 & 0,64101 & 0,99842 \\
\hline 07 & 0,72372 & 0,99389 & 0,64076 & 0,99881 \\
\hline 08 & 0,71929 & 1,00000 & 0,64000 & 1,00000 \\
\hline 09 & 0,71269 & 1,00926 & 0,63794 & 1,00322 \\
\hline 10 & 0,70513 & 1,02008 & 0,63382 & 1,00974 \\
\hline 11 & 0,69661 & 1,03256 & 0,62763 & 1,01970 \\
\hline 12 & 0,68713 & 1,04680 & 0,61938 & 1,03329 \\
\hline 13 & 0,67669 & 1,06296 & 0,60905 & 1,05081 \\
\hline 14 & 0,66529 & 1,08117 & 0,59666 & 1,07264 \\
\hline 15 & 0,65293 & 1,10164 & 0,58220 & 1,09928 \\
\hline
\end{tabular}

de ganho de peso apresentou declínio suave. Os fatores de correção foram calculados como variável contínua, mas para efeito de apresentação na Tabela 3 foram divididos em classes de 10 em 10 dias para a idade do bezerro e a data de nascimento.

Em princípio, a idade do bezerro havia sido definida como Polinômio Segmentado Quadrático-Linear, com o ponto de junção entre estes dois segmentos ("Nó") estimado aos 180 dias. Esta definição baseouse no diagrama de pontos, por inspeção e por meio de testes de experimentação, para encontrar o ponto cuja a soma de quadrados do resíduo (SQR) fosse minimizada, conforme descrito anteriormente para o Polinômio Segmentado Quadrático-Quadrático. No entanto, quando se definiu o modelo completo (com todos os efeitos, ambientais e genéticos), no qual o efeito da idade do bezerro ao desmame foi definido como Polinômio Segmentado Quadrático-Linear ou como Polinômio Quadrático Ordinário, a soma de quadrados do resíduo (SQR) e o $\mathrm{R}^{2}(0,771192)$ mantiveram-se constantes.

Da mesma maneira que o efeito da idade do bezerro ao desmame, a data de nascimento no calendário juliano, em princípio, foi modelada como Polinômio Segmentado Quadrático-Quadrático, que é uma função composta por dois segmentos quadráticos, com o ponto de junção ("Nó") estimado aos 300 dias.

Esta função foi escolhida com base no diagrama de pontos por inspeção e por meio de testes contínuos, para encontrar o ponto cuja soma de quadrados do resíduo (SQR) fosse minimizada.

No entanto, quando se incluiu no modelo completo (todos os efeitos ambientais e genéticos) este efeito como polinômio quadrático-quadrático, ocorreram problemas com o efeito da idade do bezerro - houve inversão total - ou seja, o ganho médio diário do bezerro mudou de sentido e passou a aumentar com o aumento da idade do bezerro.

A correlação entre os resíduos das covariáveis IDB e DJN, depois de removido o efeito do grupo contemporâneo, foi de $-1,00$ com $\mathrm{P}<0,001$, indicando problemas de multicolinearidade (Hoffmann e Viera, 1977, citados por PAZ, 1997). O coeficiente de correlação entre estas duas covariáveis foi de $-0,60$. Este alto valor da correlação entre os resíduos destas duas covariáveis não causou surpresa, já que a modelagem destes dois efeitos foi muito difícil, mostrando estreita relação entre a idade do bezerro ao desmame e a data juliana de nascimento. Dessa forma, estas duas covariáveis não puderam ser tratadas independentemente; por isso, o cálculo dos fatores de correção para os efeitos da idade do bezerro ao desmame e da data juliana de nascimento sobre o ganho médio diário de peso pré-desmame, com base na distribuição conjunta destas covariáveis.

Assim, procurando reduzir o correlação múltipla entre as colunas de X (Hoffmann e Viera, 1977, citados por PAZ, 1997), optou-se por modelar o efeito da idade do bezerro no desmame, como regressão quadrática (Polinômio Ordinário), e o efeito da data juliana de nascimento, como regressão linear simples.

Os coeficientes de regressão encontrados para a idade do bezerro foram: $-0,00264145 \mathrm{~kg} / \mathrm{dia}$ e $-0,00000101 \mathrm{~kg} / \mathrm{dia}^{2}$, respectivamente, para os coeficientes de regressão linear e quadrático e o intercepto $\left(b_{0}\right)$ foi 2,2510444 .

Estes resultados estão em concordância com os resultados de FRIES (1984), WOODWARD et al. (1989) e WOLF (1996), que mostraram a não linearidade do efeito da idade do bezerro ao desmame sobre o ganho de peso pré-desmame, e mais ainda 


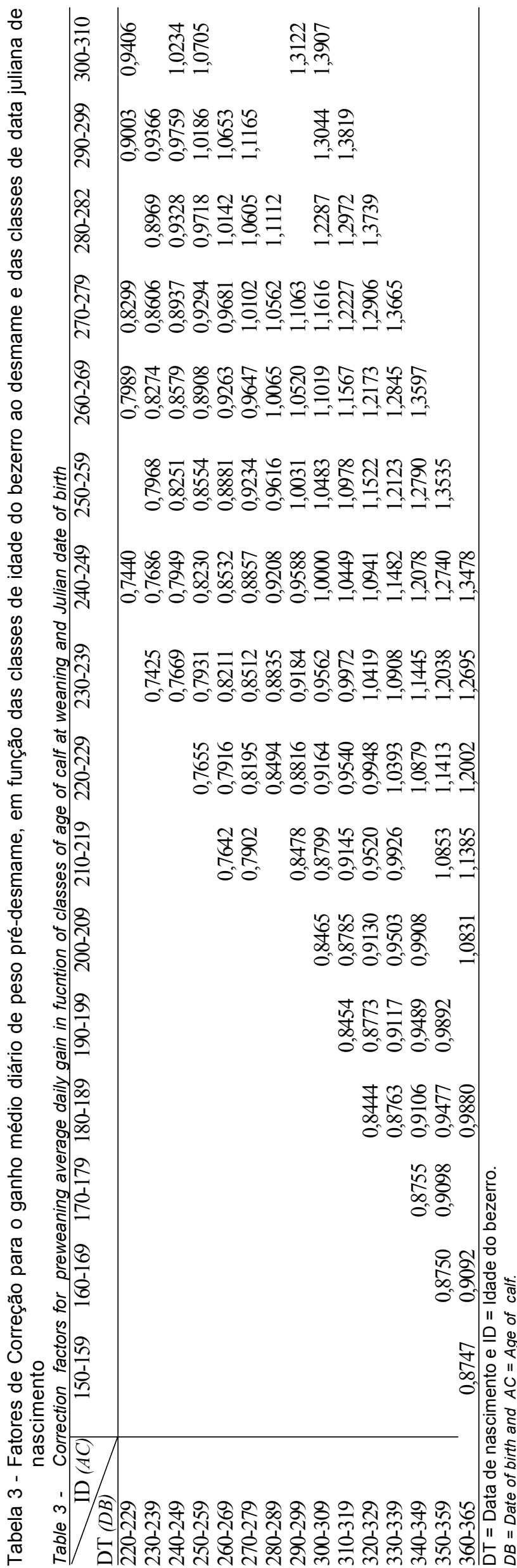

sobre o peso a desmama.

O efeito da data juliana de nascimento apresentou coeficiente de regressão linear de -0,00292770 $\mathrm{kg} /$ dia e o intercepto $\left(\mathrm{b}_{0}\right)$, de 2,2510444 . O ganho médio diário pré-desmame observado diminuiu no período de 220 até 365 dias no calendário juliano e variou de 0,75628 a $0,65378 \mathrm{~kg} / \mathrm{dia}$, correspondendo às médias das classes $11^{\mathrm{a}}$ e $18^{\mathrm{a}}$, respectivamente.

O ganho médio diário pré-desmame predito, em função da data juliana de nascimento representada por regressão linear simples e em função da idade bezerro ao desmame representada por regressão quadrática, é apresentado na Figura 2.

Os fatores de Correção para ganho médio diário de peso pré-desmame, em função das classes (de 10 dias) de idade do bezerro ao desmame e das classes (de 10 dias) de data juliana de nascimento, são apresentados na Tabela 3 .

Estes resultados evidenciam a recomendação mais recente de alguns autores (CAMPOS et al., 1989; LUCERO et al., 1995 e WOLF 1996) para o ganho de peso pré-desmame, quanto ao cálculo de fatores de correção para o efeito da data de nascimento, de que é preferível a simples inclusão do mês de nascimento no modelo de análise.

Os fatores de correção variaram de 0,7425 (classe 230-239 dias de idade do bezerro e classe 230-239 dias no calendário juliano, que representa 18 a 27 de agosto no calendário gregoriano) a 1,3907 (classe 300-310 dias de idade do bezerro e classe 300-309 dias no calendário juliano, que representa 27 de outubro a 5 de novembro no calendário gregoriano). Esta variação representa diferenças de até 39\% nos fatores de correção para os efeitos de idade do bezerro e data juliana de nascimento, calculados conjuntamente.

As diferenças superiores a $20 \%$ ocorreram nos pontos mais extremos, em que o número de observações era muito pequeno. Talvez se os nascimentos e as idades ao desmame dos animais estivessem melhor distribuídos, estas diferenças pudessem ser inferiores e a correlação entre estes dois efeitos fosse menor. Neste caso, os efeitos da idade do bezerro e da data de nascimento poderiam ser trabalhados independentemente, possibilitando o cálculo de fatores de correção para cada um destes efeitos.

Como recomendação prática, no caso destes pontos mais extremos com pequeno número de observações (animais que nascem e são desmamados nas classes com pequeno número de observações), talvez fosse melhor utilizar os fatores de correção dos pontos próximos com maiores números de observações. 
Fatores de correção para estes efeitos, estimados como função contínua e para cada rebanho, são preferíveis à simples correção linear do Peso à Desmama para o efeito da idade do bezerro ao desmame, conforme recomendado pela BIF, e à simples inclusão do mês de nascimento no modelo de análise. Neste conjunto de dados, se fosse efetuado apenas o ajuste linear para a idade do bezerro conforme a recomendação da BIF, ocorreriam diferenças de até $102 \mathrm{~kg}$ no peso ao desmame dos bezerro entre as classes de maior taxa de ganho de peso (ID=23 e DT=23) e menor taxa de ganho de peso (ID=30 e DT=30), conforme a Figura 2, o impacto destas diferenças sobre a avaliação genética dos animais deve ser considerado.

Como recomendação, para que se tenha maior precisão nas estimativas destes fatores de correção, os dados devem estar melhor distribuídos com relação ao nascimento, minimizando os possíveis problemas de multicolinearidade e/ou concentração dos dados. No entanto, como recomendação ao criador, pode-se sugerir a necessidade de se controlar o grande intervalo de nascimento na tentativa de agrupar os animais nos pastos e grupos de manejo, conforme a data de nascimento procurando concentrar os nascimento nos períodos em que se obtêm as taxas de ganhos de pesos superiores.

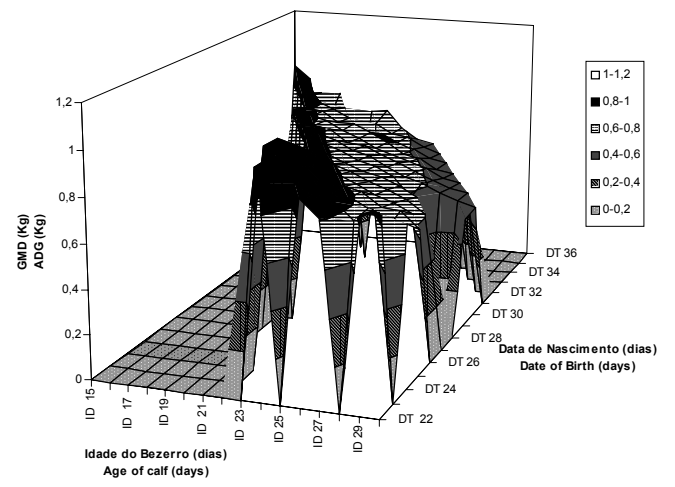

Figura 2 - Ganho médio diário pré-desmame predito, em função da data juliana de nascimento (DT), representada por regressão linear simples, e em função da idade bezerro (ID), representada por regressão quadrática.

Figure 2- Preweaning average daily gain according of Julian birth date represented for linear regression and according age of calf represented for quadractic regression.

\section{Conclusões}

Os resultados do presente trabalho sugerem que o ajuste linear para a idade do bezerro ao desmame não elimina completamente este efeito, mesmo dentro de intervalo de 90 dias. Fatores de correção para os efeitos de idade do bezerro e data juliana de nascimento, estimados como função contínua, devem ser utilizados preferencialmente à simples inclusão de mês de nascimento no modelo de análise. Outros fatores de ajustes para os efeitos da idade da mãe e idade do bezerro (nãolinear), além das recomendações internacionais, podem ser utilizados para tornar a seleção mais precisa.

\section{Agradecimentos}

Ao Dr. Antonio Batista Sancevero e à Granja Rezende S. A., pelo fornecimento dos dados, e ao Dr. Vanerlei Mozaquatro Roso, pela pré-edição dos dados.

\section{Referências Bibliográficas}

AHUNU, B., MAKARECHIAN, M. 1986. Influence of birth date, sex of calf, breed group and age of dam on preweaning performance of range beef calves. Can. J. Anim. Sci., 66(2):381-388.

AHUNU, B., MAKARECHIAN, M. 1987. Preweaning patterns of growth in three breed groups of range beef calves. Can.J. Anim. Sci., 67(3):653-661.

ANDRADE, A. B. F., PAZ, C. C. P., FARO, L. E. et al. Estimativas de parâmetros genéticos e fenotípicos dos pesos ao nascimento e à desmama e do ganho de peso pré-desmama em um rebanho Canchim. In: REUNIÃO ANUAL DA SOCIEDADE BRASILEIRA DE ZOOTECNIA, 31, 1994, Maringá. Anais ... Maringá: SBZ, 1994. p.158.

BIF. Guidelines for uniform beef improvement programs. Raleigh: U. S. Dept. Agriculture - North Carolina State University, 1996, 155p.

BOLDMAN, K.G., KRIESE, L.A., VAN VLECK, L.D. et al. A manual for use of MTDFREML. A Set of Programs to Obtain Estimates of Variances and Covariances. U. S. Service, 120p. 1993.

CAMPOS, L.T., SILVA, P.R., FRIES, L.A. 1989. Fatores de correção para efeitos ambientais que afetam o ganho do nascimento à desmama em bovinos da raça Nelore. Coletânia de Pesquisas Inéditas sobre Zebu. Empresa de Pesquisas Agropecuária de Minas Gerais. Uberaba, MG. p.108-123.

CARDEllino, R. A., CASTRO, L. F. S. 1987a. Efeitos ambientais e genéticos e fatores de correção para peso ao nascer, peso à desmama e ganho de peso pré-desmama, em bovinos Nelore. R. Soc. Bras. Zootec., 16(1):14-27.

ELZO, M. A., QUAAS, R. L., POLLAK, E.J. 1987. Effects of age of dam of weight traits in the Simmental population. $J$. Anim. Sci., 64(4):992-1001.

EUCLIDES FILHO, K., NOBRE, P. R. C., ROSA, A. 1991. N. Idade da vaca e suas inter-relações com a fazenda, reprodutor e sexo do bezerro. R. Soc. Bras. Zootec., 20(1):40-46.

FRIES, L. A. A study of weaning weights in Hereford cattle in the state of Rio Grande do Sul, Brazil. Ph.D. Thesis. Iowa State 
Rev. bras. zootec.

University, Ames, Iowa, 1984.

FULLER, W.A., 1969. Grafted polynomials as approximating fuctions. Austr. J. Agric. Econ., 13:35-46.

LUCERO, C.E., CAMPOS, L.T., CARDOSO, F.F. CARDELLINO, R.A., DIONELO, N.J.L. Fatores de correção do ganho de peso pré-demama, pela idade da mãe e do bezerro, e data de nascimento, na raça Nelore. In: REUNIÃO ANUAL DA SOCIEDADE BRASILEIRA DE ZOOTECNIA, 32, 1995, Brasília, DF. Anais... Brasília: SBZ, 1995. p.616-618

MILLER, P. 1973. A recent study of age adjustiment. J. Dairy Sci., 56(7):952-958.

PAZ, C.C.P. Efeitos ambientais e genéticos que afetam o ganho de peso pré-desmame em bovinos da raça Nelore. UNESP, Jaboticabal, SP. 1997. 117 p. Dissertação (Mestrado em Ciências Veterinárias) - Universidade Estadual de São Paulo, 1997.

PEIXOTO, A. M. 1993. Fatores que interferem no crescimento do gado de corte até a desmama. In: Bovinocultura de Corte. Fundamentos da Exploração Racional. p.129-158. Piracicaba: FEALQ (550p).
SAS User's Guide: Statistical Analysis Systems. Cary, 1992.

SOUZA, J. C., BRULÉ, A. O., FERRAZ FILHO, P. B. 1994. Repetibilidade dos pesos e ganho de peso do nascimento à desmama de bovinos da raça Nelore. R. Soc. Bras. Zootec., 23(1):133-139.

WOLF, P. G. L. Efeitos da heterose individual e materna sobre o ganho de peso do nascimento ao desmame de terneiros Pampiano-Braford. Porto Alegre, 1996. 114p. Dissertação (Mestrado em Ciências Veterinárias) - Faculdade de Veterinária, Universidade Federal do Rio Grande do Sul, 1996.

Recebido em: $13 / 03 / 98$

Aceito em: 10/08/98 